\title{
Virtualizing Monitoring and Control Systems: First Operational Experience and Future Applications
}

\author{
Michael Schmidhuber ${ }^{1}$, Ursula Kretschel ${ }^{2}$, Thomas Singer $^{3}$ \\ German Space Operations Center, DLR Oberpfaffenhofen, Germany \\ Andreas Uschold ${ }^{4}$ \\ Technical University of Munich, Germany
}

\begin{abstract}
Virtualization is a technology that allows emulating a complete computer platform. The potential use ranges from consolidating hardware to running several different operating systems in parallel on one computer to preserving the operability of heritage software. GSOC has been investigating the possibilities of virtualization for some time. Aside from the usual approach of virtualizing the central servers out of administrational, consolidational reasons, the possibilities and advantages of control room client virtualization was explored. While moving mainstream in other businesses, the space community is cautious to apply this technique to the mission critical monitoring and control systems. This paper illustrates three virtualization steps that are underway at GSOC and presents the experiences gained.
\end{abstract}

\section{Nomenclature}

$D L R=$ German Aerospace Center

GSOC = German Space Operations Center (part of DLR)

TSX $=$ TerraSAR-X

$T D X=$ TanDEM-X

$V M \quad=$ Virtual Machine

$O / S \quad=$ Operating System

$V L A N=$ Virtual LAN (local area network)

LEOP $=$ Launch and early orbit phase

$M \& C=$ Monitoring and Control

AOCS $=$ Attitude and Orbit Control (Subsystem)

$T M \quad=$ Telemetry

\section{Introduction}

$\mathrm{V}$ irtualization is a technology that has become widely accepted in a large variety of businesses and applications. It emulates a complete computer platform and allows running it as an application on a host computer.

An increasing number of new and concurrently running projects at the German Space Operations Center (GSOC) require more flexible and short-term adjustments of the operational environments.

Virtualization is a technology that has rapidly evolved in the last years as more powerful and advanced hardware has become widely available. It allows emulating a complete computer platform. The potential use ranges from consolidating hardware to running several different operating systems in parallel on one compute to preserving the operability of heritage software.

GSOC has been investigating the possibilities of virtualization for some time. Aside from the usual approach of virtualizing the central servers out of administrational, consolidational and redundancy reasons, the possibilities and advantages of control room client virtualization was explored.

${ }^{1} 82230$ Wessling, Germany; michael.schmidhuber@dlr.de

282230 Wessling, Germany; ursula.kretschel@dlr.de

${ }^{3} 82230$ Wessling, Germany; thomas.singer@dlr.de

${ }^{4}$ Arcisstrasse 21, 80333 Muenchen, Germany; andreas.uschold@mytum.de 
Client virtualization offers the potential to reduce the long-term blocking of control rooms during the system build-up phase and to increase the flexibility of the installed system.

While moving mainstream in other businesses, the space community is cautious to apply this technique to the mission critical monitoring and control systems.

This paper illustrates two virtualization projects that are underway at GSOC and presents the experiences gained.

The mission TanDEM-X that will be launched in May 2010 offers a unique opportunity. The operations scenario of this mission includes dual satellite operations with TerraSAR-X in very close formation. This demands to monitor both spacecraft at the same time from the same control room by largely the same team. It was decided to explore the way of virtualizing the Monitoring of the TerraSAR-X spacecraft on top of the TanDEM-X installations.

The ground data systems group is under pressure to ensure a strict configuration control on a control room system which is permanently in use while allowing modifying and updating parts of it permanently. Virtualization based installation is being introduced to allow reaching this goal.

\section{What is Virtualization?}

Several meanings of the term virtualization have been known over the history of computer development. They must not be mixed up. Other contemporary meaning are hardware virtualization, where e.g. several hard disk drives are combined into a large "virtual" disk, or network virtualization where physical network systems are combined or divided into virtual network like a VLAN. The meaning being discussed in this paper and that is the most commonly understood today, is the software emulation of entire computer platforms.

Virtualization means the complete abstraction of a computer. A virtual machine executor allows configuring a complete environment and setup for the virtual machine. It provides simulated CPUs, mass memory, hard disk drives, network adapters and other interfaces within the memory space of the VM executor. From the perspective of the host operating system, the VM is totally encapsulated within the VM executor process. Additionally only the external interfaces are visible. Access to host resources is handled like for any other concurrently running applications. The VM Executor then allows installing an O/S fitting its architecture. No special configurations or drivers are necessary. The completely installed guest $\mathrm{O} / \mathrm{S}$ can then be treated just like any other physical computer. Neither the guest system nor the applications running can distinguish if they are running virtually or not. Guest application or system crashes typically do not affect the host $\mathrm{O} / \mathrm{S}$. The exception here is the resource usage on the host computer.

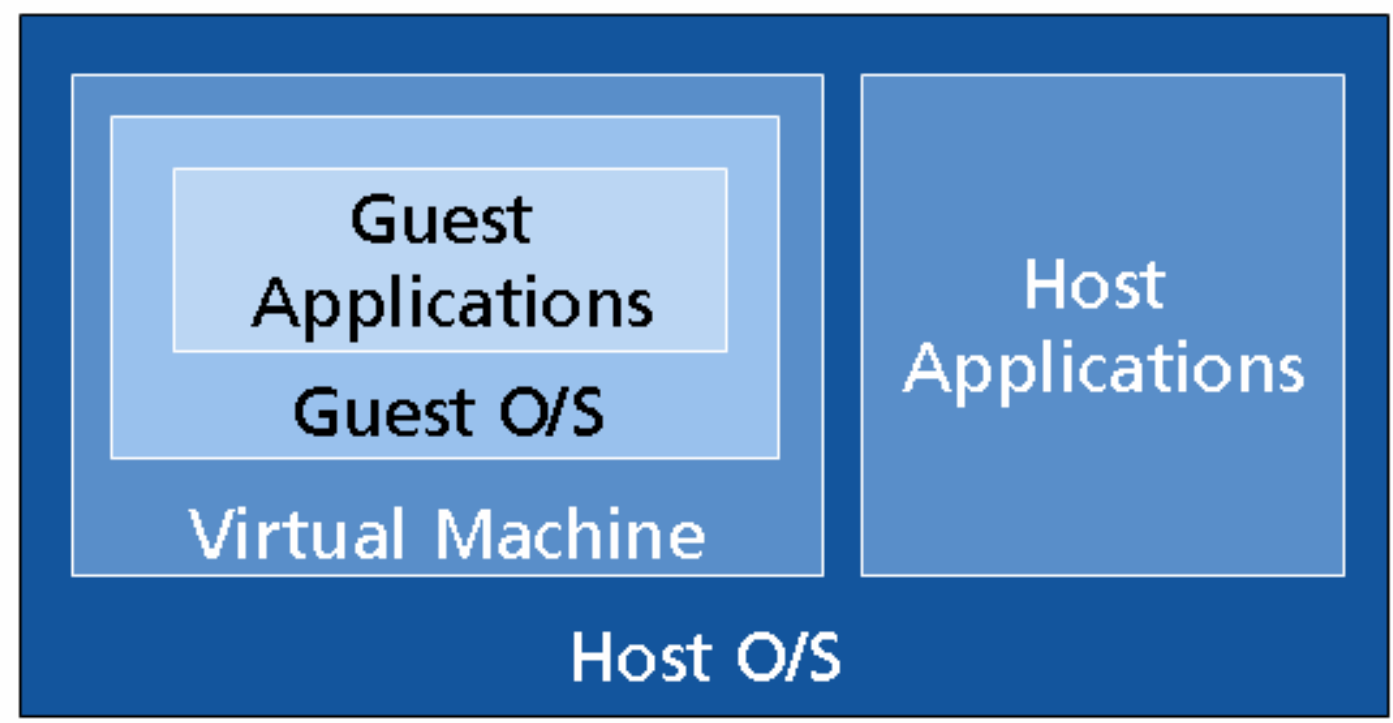

Figure 1: The principle of virtualization.

Limitations exist in that not for all platforms and O/Ses virtualizations are available. Also the variety of virtualized hardware components is limited. Huge gains in virtualizability came from the introduction of hardware abstraction layers in device driver development. Therefore especially older hardware is more difficult to virtualize. 


\section{The Potential of Virtualization}

The applications for virtual machines can be divided into three parts. The first is the hosting aspect. It becomes possible to locate two or more different $\mathrm{O} / \mathrm{S}$ on one platform during runtime, thus reducing the need for additional hardware. Applications that are easy on resources can be isolated from each other and still be located on the same host. Environments can be preserved that are dependent on an older $\mathrm{O} / \mathrm{S}$ for which hardware can be no longer procured. This is also important for reference or development systems that can be stored for longer periods without occupying room and consuming energy. The second category is the persistence aspect. Work on a virtual machine can be suspended at any time and resumed later or even be transported to a different host (provided that it is equipped with a compatible VM executor). The complete operational state of the machine is stored in a single file on the host. This includes the memory content and the running applications. The same VM can be stored at different times, reflecting different stages of system installation or application progress. Programs become pausable and resumable which have never been designed for this. The third area of virtualization is the configurational aspect. It suddenly becomes very easy to reset a system to a defined status in a matter of seconds without resorting to clumsy methods like disk images.

\section{Dual Installations for TSX/TDX operations}

\section{A. Operations Scenario “Dual Satellite Operations”}

TanDEM-X is a two satellite mission in close formation. The spacecraft are on the same orbit and only separated by variations in the eccentricity and inclination of the individual orbits. This results in a so-called helix orbit, where the two spacecraft are revolving around each other once per orbit. Their distance varies between 200 and 600 meters. This scenario leads to operational constraints, as they are always at the same time in view from the same ground stations. Also the maintenance of the correct orbit separation is an important point, as the safety could be endangered by spacecraft collisions or radar beam disturbances. Therefore tight and coordinated control over both spacecraft is necessary especially during parts of the commissioning phase.

GSOC is intending to approach this problem by a collocation of the operations of both spacecraft from the same control room with the same team. TSX is in routine operation since June 2007 and is operated from the K9 multimission control room of GSOC. Operations during the LEOP of TDX will be done from the K2/K2a control rooms. These rooms are a lot larger in order to provide space for more than 22 console positions.

During LEOP all positions will be occupied by operations engineers, scientist and the support team from the manufacturer. Already at each position, there are three computers and three to four monitors. It is not possible to set up a complete second system for each operations console. Commissioning will see a slight reduction in staffing, however space is still too constrained and it is not desired to start extensive reconfiguration work during this early phase.

\section{B. M\&C System Components}

GSOC currently uses Shuttle XPC systems (small form factor) with $1.6 \mathrm{GHz}$ dual core CPU (6320 chip set) with 2 GByte memory. They are equipped with a dual graphics interface that is mostly connected to one monitor only due to space contraints. A the monitor exit is connected to a neighboring monitor on its secondary input. This allows to expand the available monitoring area selectively.

SCOS2000 is the prime M\&C component for TDX and TSX. It is developed and provided by ESA. It is running on LINUX as an operating system. GSOC uses a modified version of the 3.1 release of SCOS2000. Originally this needs LINUX 8.2 for which no more hardware is available. GSOC has established a way to run SCOS also on SLES10. Note: this problem could have been another example for the use of virtualization.

For TM monitoring the software SATMON is used however. This application was developed by Heavens-Above for GSOC. It connects to SCOS and displays the already processed telemetry in a convenient and flexible way. The software is based on Windows and .NET as operating system. GSOC uses Windows XP Professional SP2 and .NET 3.5 for this task. 


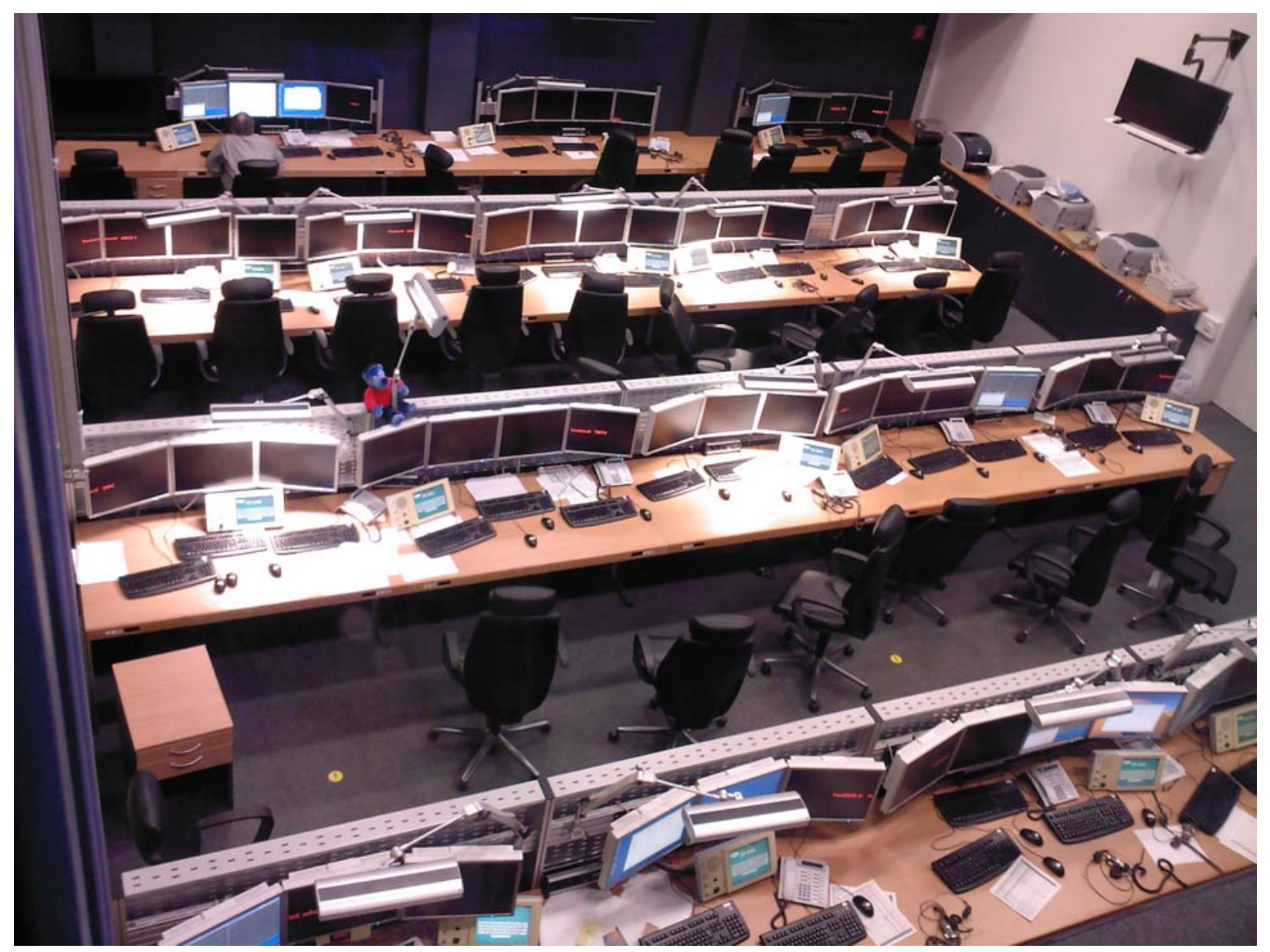

Figure 2: The LEOP and commissioning control room K2 for TDX. During commissioning the TSX spacecraft is operated in dual satellite operations mode.

\section{Fitting Two M\&C Systems Into One Console}

With already three computers in one console cabinet and the accompanying effort in cabling and thermal load putting up a separate PC for TSX monitoring does not seem a feasible solution. Not to mention the fourth keyboard and mouse.

SCOS2000 in its release 3 does not permit several instances of SCOS to run at the same time on one computer. Simultaneous running is however desired to permit fast switching of focus between satellites.

Virtualization offers a near ideal solution for this. While running one SCOS (TDX) on the PC directly, a second SCOS is encapsulated in a virtual machine and configured for TSX. In this way both SCOS can be running on one PC at the same time. The virtual machine has its own IP address and also in other aspects behaves independent from the host. E.g. a complete new login is needed when the VM has been started and also when SCOS is launched. To make it easier to keep the projects apart different colors were used for the desktop background. This color scheme was extended also to other tools like SATMON (TM monitoring) and the OPSWEB intranet.

The critical positions "Command" and "AOCS" were equipped conventionally with two PC systems and additional monitors. The SATMON TM monitoring application natively allows running two instances at the same time that are connected to different servers / projects. 


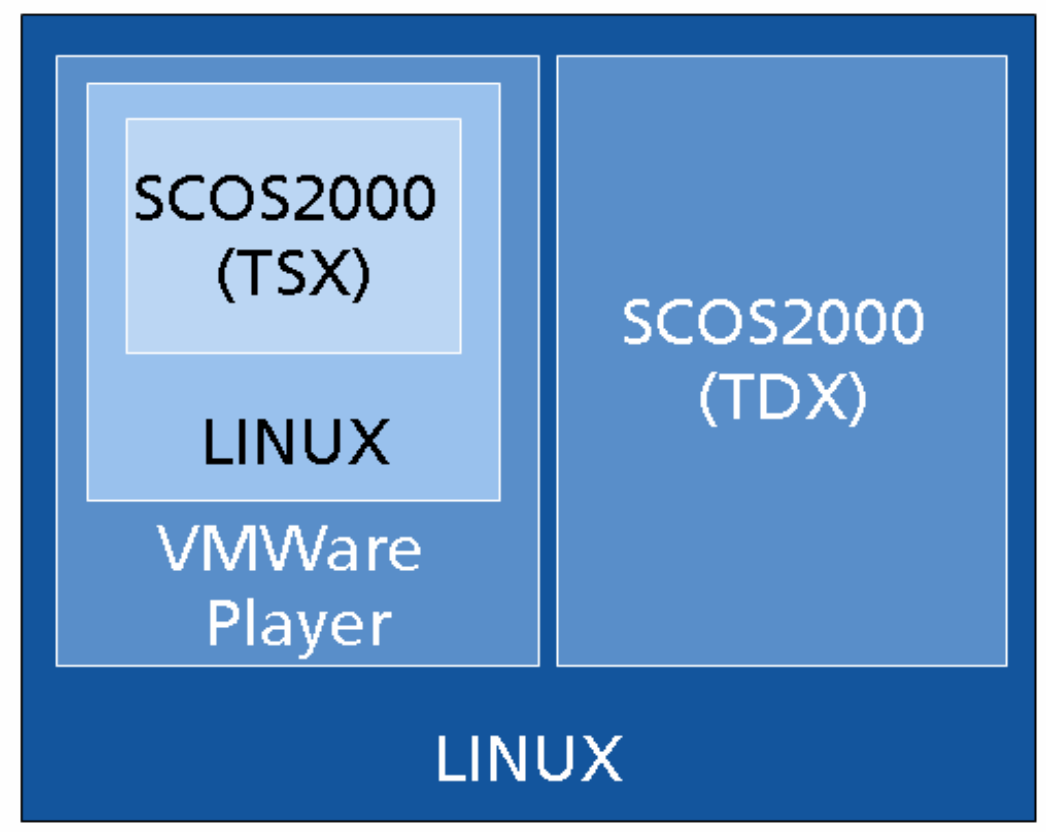

Figure 3: TheVM setup of the dual satellite workstation for TSX/TDX

\section{Performance}

Modern PC hardware has experienced a major leap in suitability for virtualization after the introduction of multicore CPUs. The operating systems and the applications originate from several years in the past where resources and processing power was still a lot lower. This results in a situation where enough idle resource is available on the console to house two projects. Tests and the use in mission simulations showed that the usability is high and close to using two separated systems. Switching between host and guest application is easy and fast. No resource conflicts were observed (e.g. network bottleneck). The stability is also without any negative experience that could be traced to the virtualization itself.

The only aspect that is not solved by this setup is the constrained monitor area. Even for one project, this resource is already limited. On selected consoles, it was solved by adding a fourth monitor or making monitor inputs switchable to add area when needed.
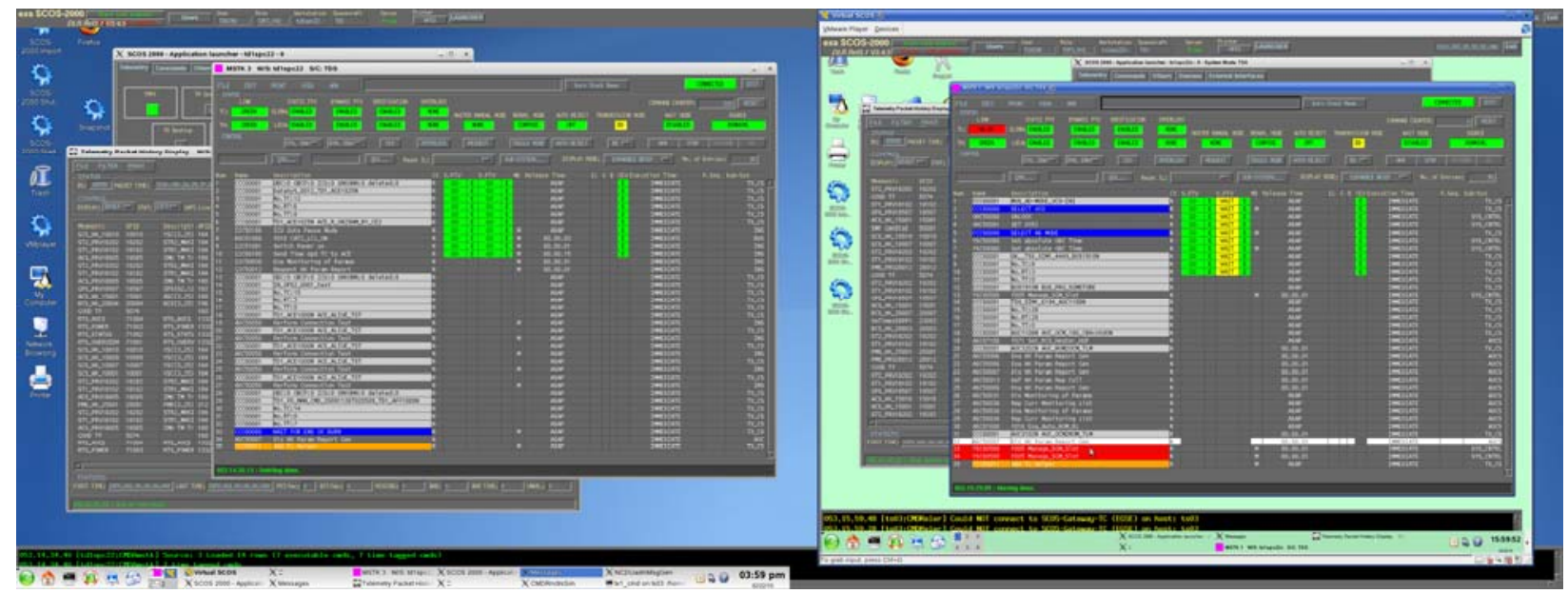

Figure 4: Two SCOS instances running on a single PC with two monitors. The TDX SCOS is spanning the complete screen (dark blue with SCOS header and footer). The SCOS windows on the left part of the screen are dedicated to TDX. The virtual machine can be seen on the right part. It displays another LINUX desktop in light green, and shows the TSX SCOS header, footer and manual stack windows. 

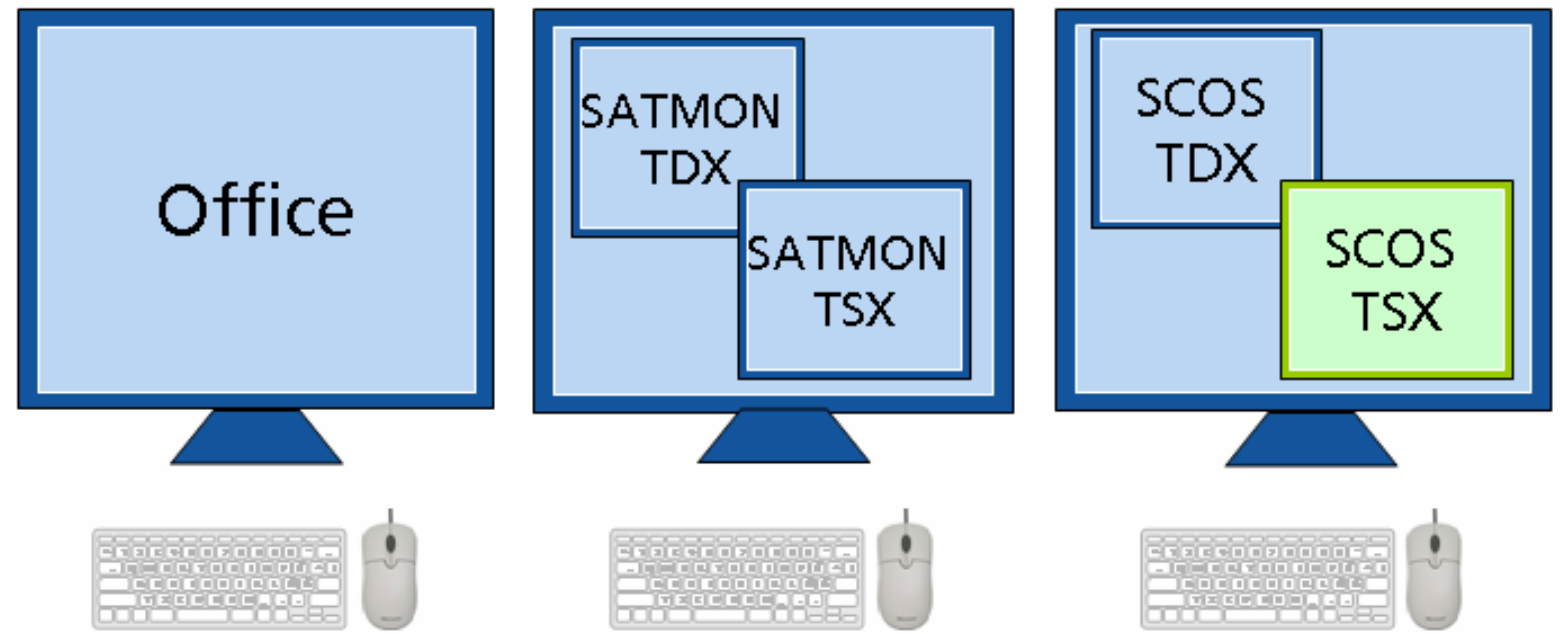

Figure 5: Console layout for dual satellite operations at a three monitor console.

\section{E. Acceptance}

Virtualization is a rather new technology and space operation is a conservative and cautious business. Before the widespread use of the term "virtual", famous science fiction writer Stanislaw Lem has suggested to use the word "phantom" for the same meaning. Virtual reality has become a buzzword some time ago (termed "phantomatic" by Lem 20 years ago). It is used for displaying things that are not really happening at the place where they seem to be happening. This makes clear that the term has the connotation of things being "not real".

The project team was reluctant at first to accept this method. With a clear emphasis on an unmodified host setup for TDX LEOP operations, it was possible to convince them into a trial in a real mission rehearsal. In addition, it was important to distinguish between commanding and monitoring. Therefore, the positions command and AOCS were set up conventionally occupying more console space. After the mission rehearsals were performed without problems, acceptance of virtualization became considerably higher.

\section{F. Looking around}

In the mean time, other projects have not been idle. They also use dual platform systems. One example is the Columbus Control Center (also at GSOC). They use VM to run the SATMON TM monitoring software, which is only a supplement to their system, on their LINUX host PCs since two years without any problems. Also a communications satellite project employs a VM to host SATMON on SCOS machines for space constraint reasons at a remote monitoring location.

\section{G. Conclusion}

The system presented here is a valid and positive solution to the problem at hand. It has to be noted that running guest and host applications together does not fulfill the normal paradigm of virtualization. This philosophy teaches that platforms shall remain independent from each other. In the presented scenario, the TSX platform is clearly dependent on the TDX platform. Future scenarios will go different ways (cf. rest of paper). E.g. running two virtual machines on one host instead. However, for transition, building confidence and gaining experience it was a necessary, helpful and simple step.

\section{Complete Client Virtualization}

\section{A. Goals}

After the positive experiences with control room virtualization for the TSX and TDX projects, the introduction of virtualization technologies is planned for the control rooms in general which includes a multitude of projects in the GSOC MultiMission Control Center. Unlike to TSX and TDX those projects are completely independent of each other. As they need their well-defined discrete resources increased requirements result from this. The objective 
of further tests is to find a preferably stable virtualization platform which offers such functions and does not lead to large additional administrative work.

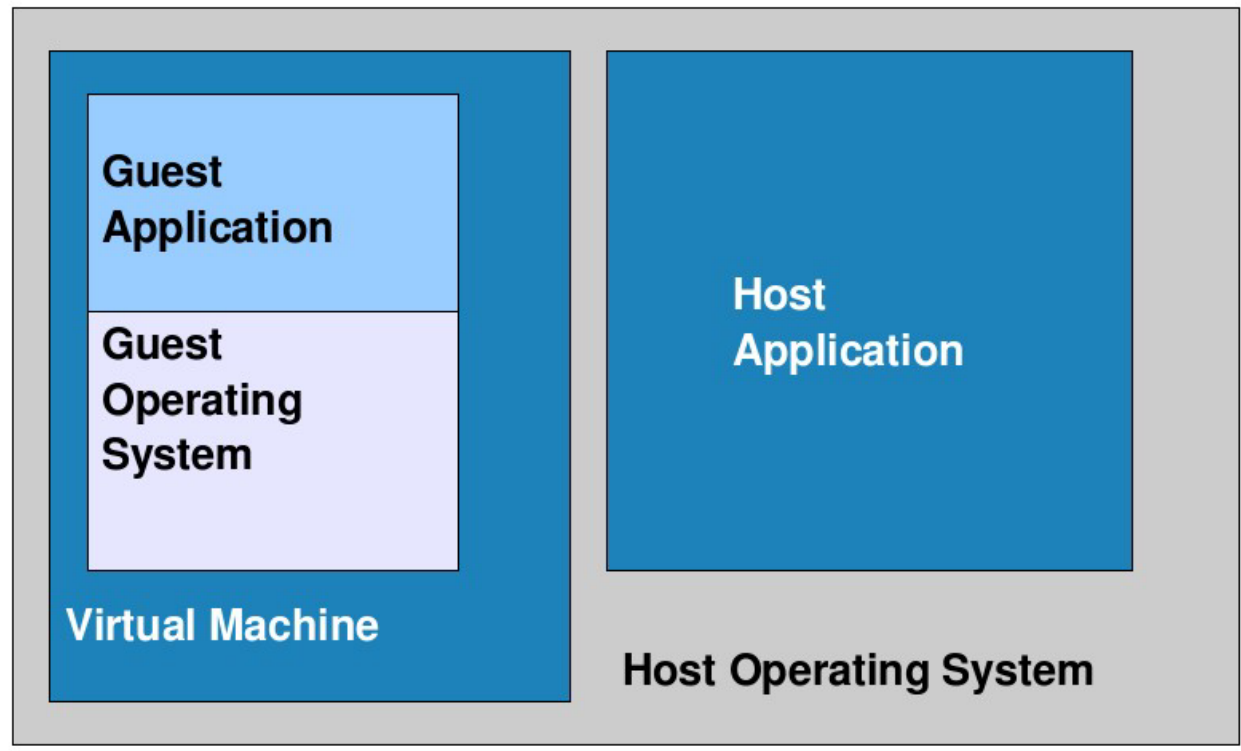

Figure 6: Host-Based Virtualization System based on X86 Architecture

\section{B. Bare-Metal Hypervisors}

In GSOC a virtualization solution providing typical server services (ftp, dns, virus scanner, etc.) has been running stable for 1.5 years. This setup is based on bare-metal hypervisors and shows that these provide the desired solid platform for virtualization.

Unlike to conventional O/Ses, hypervisors are a thin O/S layer developed especially for virtualization. On this basis, VMs are running in parallel, use strictly separated resources and can be configured independently of each other.

In addition, new VMs can be added or existing VMs can be deleted anytime without having an impact on other operational VMs.

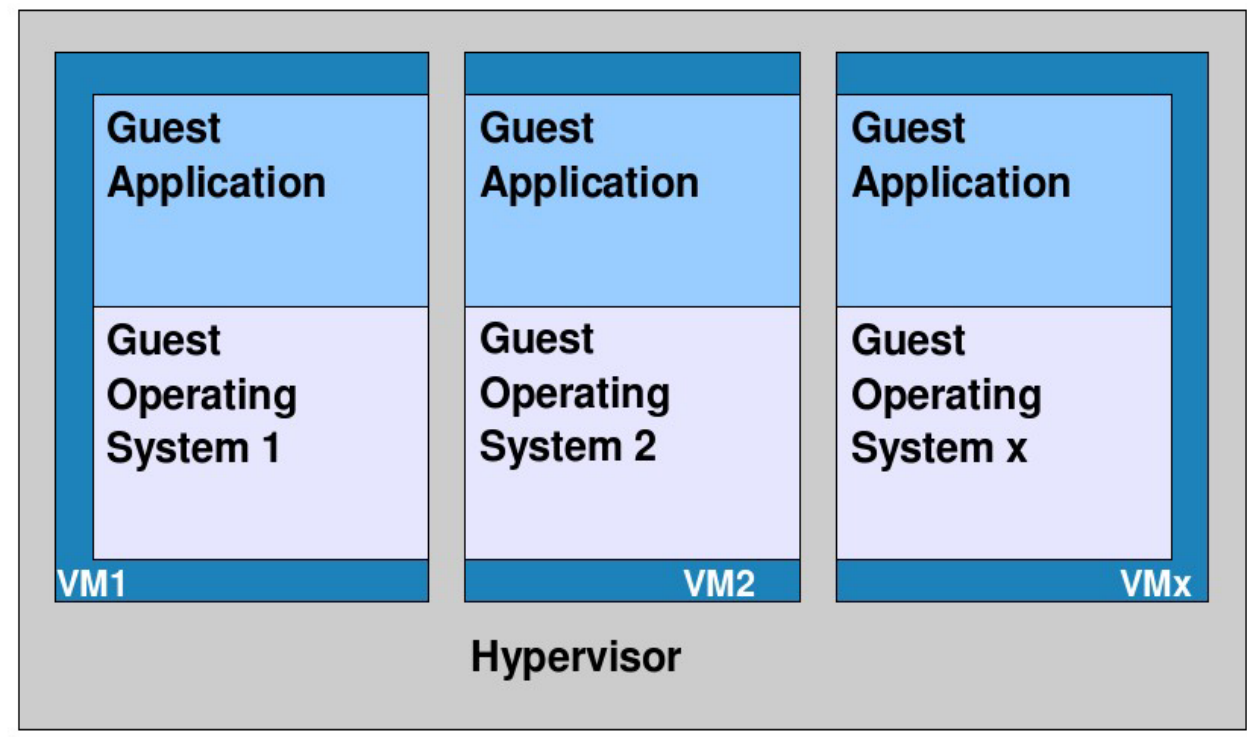

Figure 7: Bare-Metal Hypervisor based on X86 Architecture

\section{Special Requirements}


Bare-metal hypervisors only support a limited range of server hardware. Depending on the number of VMs, which will jointly be running on a single hardware, the servers have to be sufficiently equipped especially with processors, random access memory and network connections. Unlike to the virtualization solution used for the TSX/TDX projects, the contents of the running VMs can not be accessed locally but via the IP network, so that a high-capacity network infrastructure has to be in place for operations.

\section{Advantages of Bare-Metal Hypervisors}

In addition to the advantages of virtualization in general there are some points which only apply to hypervisors. As this thin O/S layer is specifically designed for virtualization, it offers a remarkably higher performance than the solution used for projects at GSOC up to now. This can be seen both in the performance of each single VM (related to processor power, I/O, network, etc.) and in the number of VMs, which can be set up on a single hardware. Thus it is possible to operate at least eight VMs, which are completely independent of each other, in parallel on one server (server hardware: 2 x Intel Xeon E5530 Quadcore, 24 GB RAM). Furthermore all VMs running on top of hypervisors can be administrated centrally with commercially available tools. Those provide a time-saving management possibility, specifically the deployment of new VMs is simplified.

\section{E. Test Installation}

A bare-metal hypervisor (VMWare ESXi) was installed on suitable server hardware connected to a test IPnetwork and configured. On this basis, several VMs with different O/Ses (e. g. Windows XP SP3, openSuSE Linux 11.2, RedHat derivatives, etc.) were set up as workstations which can then be accessed via the network. Standard workstations, laptops and thin clients that were also connected to the test IP-network acted as client systems. It showed up that it is possible with each of the mentioned systems to put the desktop of the VM provided by the server on top of the locally existing desktop. Applications which were started in the VMs were running without noticeable performance loss on the client side. As expected, several different virtualized desktops can be displayed on client systems with multiple attached monitors, without having any constraints with respect to the installed O/Ses.

In these tests, the used thin clients proved to be particularly suitable, because they are especially designed for the use of remote desktops (running on physical or virtual machines) and they are running very stable. With the preinstalled connection manager the desired connections to virtualized workstations can be edited quickly and smoothly. As a central management tool is available, a simultaneous configuration of those connections for multiple thin clients is possible. The used thin client supports high resolutions on up to six monitors, which can lead to a significant reduction of necessary hardware.

\section{F. Implementation of the „Virtualized Control Room“ for Satellite Missions}

In the following it will be shown by means of an example how a solution with virtual desktops can be applied for mission operations.
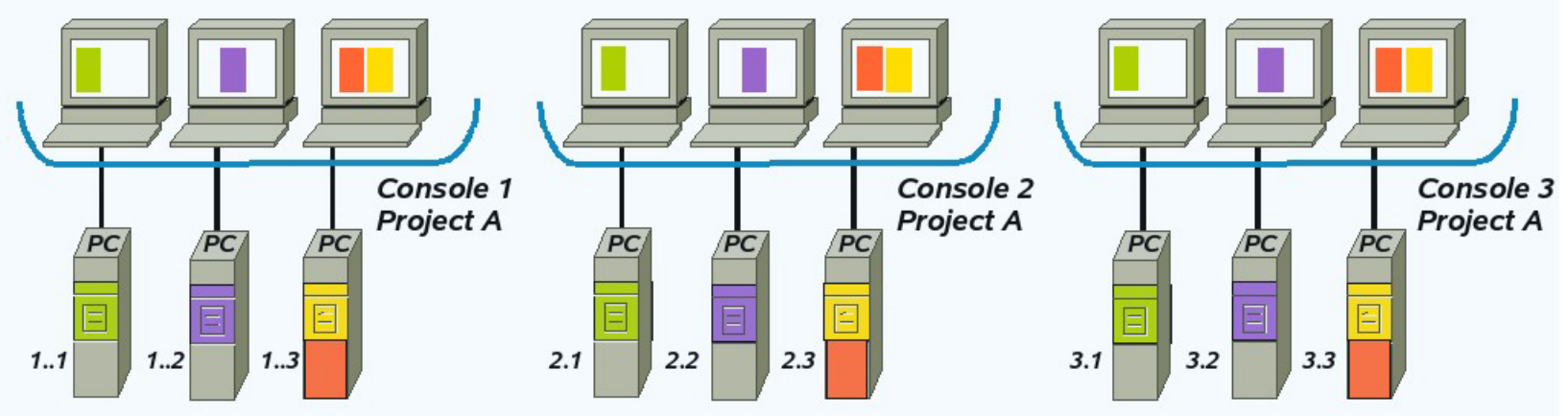

Figure 8: Current Scenario, Control Room (Workstations)

Up to now a satellite project needs for instance three workstations per console, which are used for functions like SATMON, SCOS, etc. For a control room with 20 consoles this means that for this project 60 PCs have to be integrated, installed and configured. Any modifications, e. g. for emergency support, tests and simulations also for other projects in this control room, lead to a high effort in time and administrative work, because other O/Ses, other applications or even only other software versions may be needed. 
With desktop virtualization it is now possible to replace those three workstations in one console of the control room by a simple terminal like a thin client. The functions SATMON, SCOS, etc. are provided as independent VMs on capable server hardware with installed hypervisor. Each VM is also running on a second server for redundancy reasons. In the mentioned example only installations for six VMs instead of 60 workstation installations are necessary.

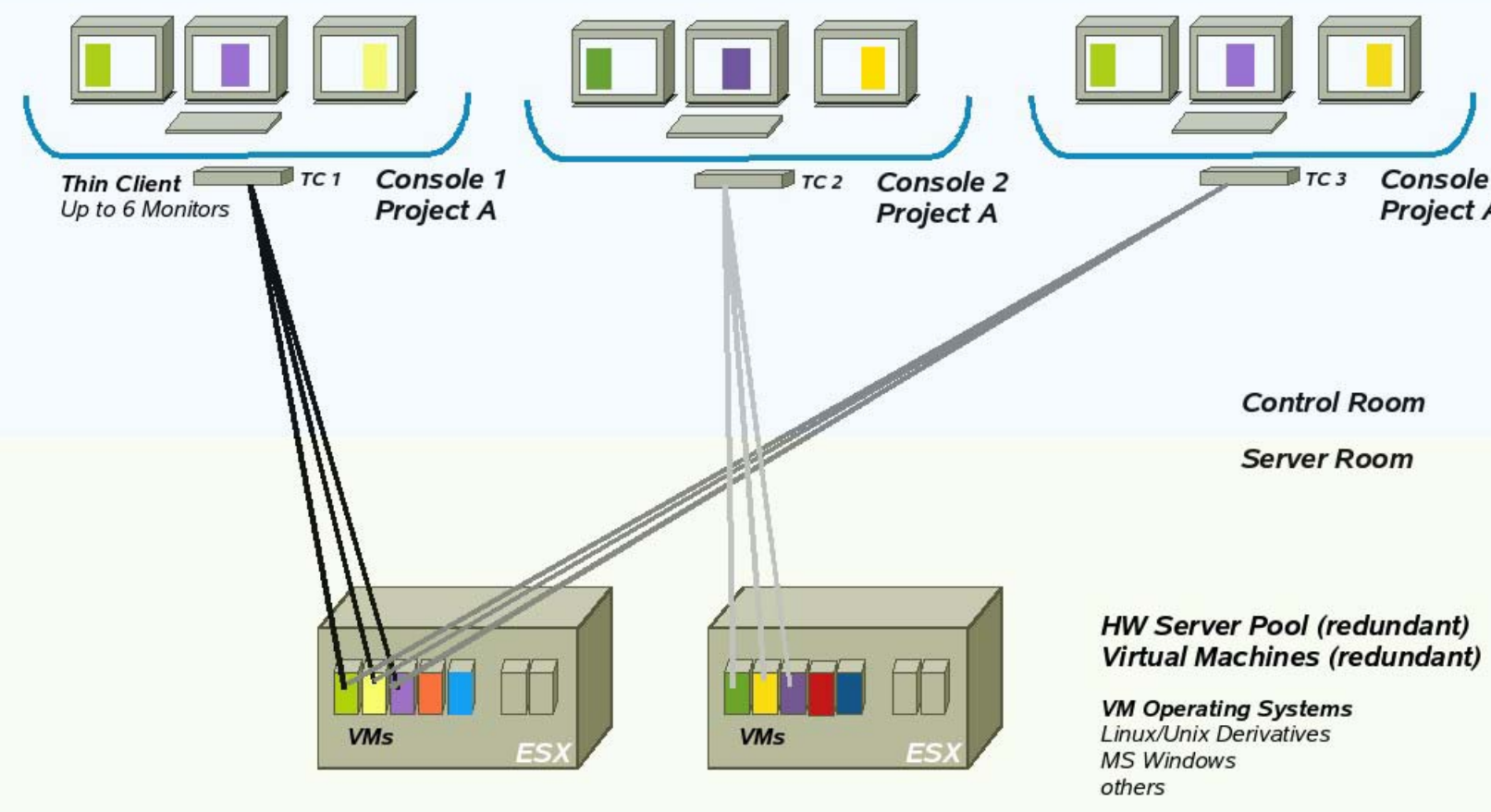

Figure 9: Virtualized Scenario (Thin Clients and Virtual Machines)

On all 20 thin clients connections to all virtualized desktops are preconfigured. By default one half of the thin clients uses the VMs on server 1, the other half the VMs on server 2. If one server (e. g. server 1) fails, fully operational consoles are still available. The consoles not serviceable at this point can also be made functional again by using the preconfigured connections to server 2 , which were not in use up to now.

For the provision of new functions simply a new VM has to be installed, which is then rolled out to two servers, as well as the necessary connections have to be configured on the thin clients. 


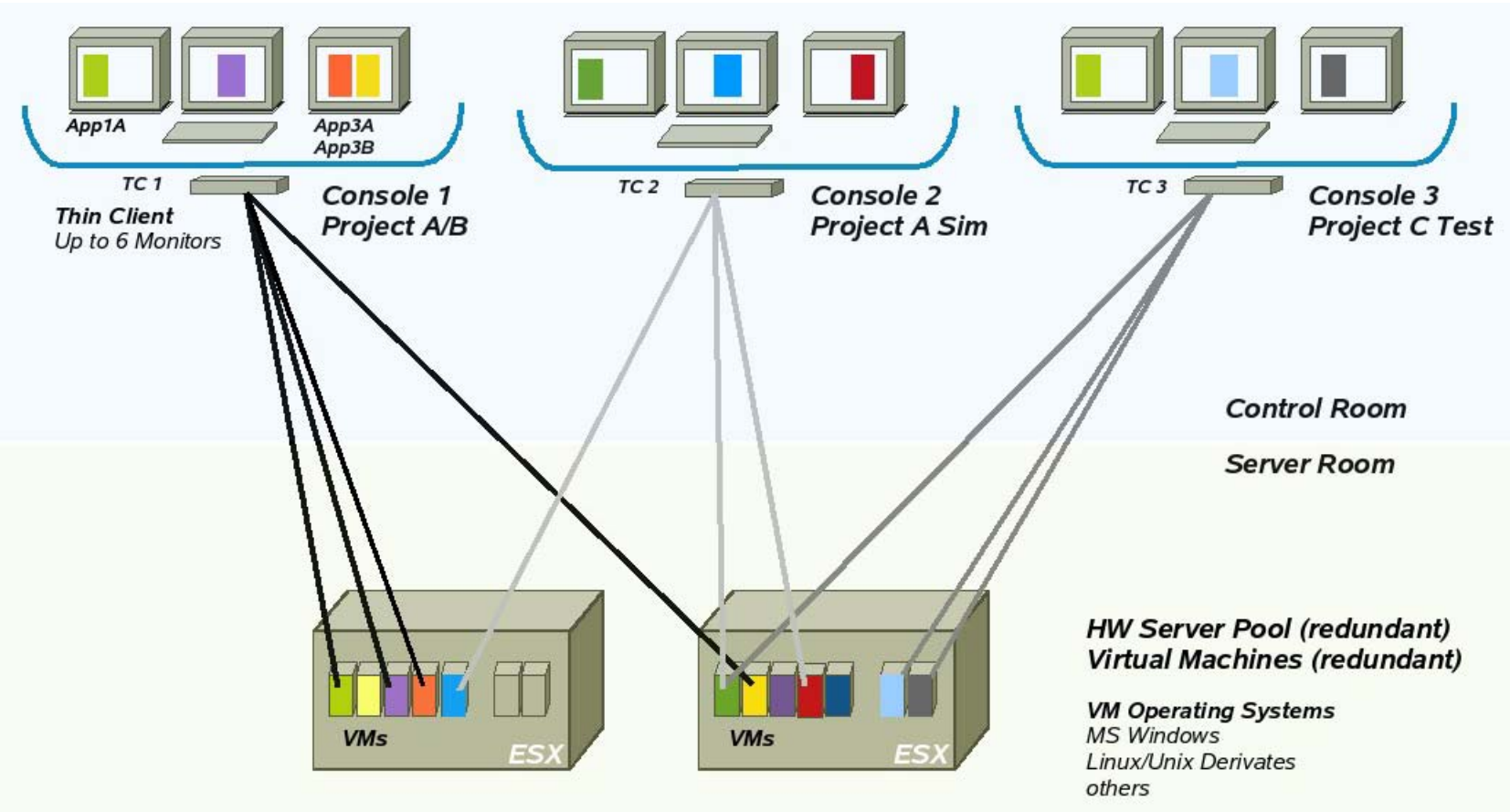

Figure 10: Virtualized Flexible Scenario (Thin Clients and Virtual Machines)

\section{G. Benefits from the „Virtualized Control Room“}

Regarding the above control room setup it involves only little effort in time to access any functions of all projects. As rarely needed functions can definitely be hold ready on servers in the form of a powered off VM, the existing resources are not needlessly loaded with this scenario. In that case the desired working environment will be available within a few minutes if necessary.

As the above named example points, the hardware needed in control rooms could be drastically reduced. Because thin clients can be used for all satellite projects and hardware failures hardly occur, the control rooms do not have to be altered between different missions and can be used in a much more flexible way. For this reason a higher degree of utilization of these rooms is possible, which is particularly valuable with respect to the increasing number of projects at GSOC.

In addition to the reduced number of necessary installations mentioned above, the administrative work is strongly simplified by the central management options available for VMs and thin clients. Furthermore the presented system can be expanded by servers and VMs anytime without restricting the usability of the control room, as such administrative actions are completely performed in the background.

Considering these points it is quite obvious that a large savings capacity exists concerning the costs for rooms, energy, hardware and administration.

\section{Conclusion}

GSOC has successfully applied virtualization in several areas of space operations. The applications illustrated above were important steps towards the larger goal of full use of client virtualization. It could be demonstrated that virtualization fulfills the requirements of space operations like stability, reliability and robustness of handling,

The primary objectives like savings in energy consumption, cost of hardware and housing were largely reached. It remains to be demonstrated that virtualization can take the full load of work for critical tasks like telecommanding. The confidence for this step will be strengthened by positive experience in the monitoring tasks.

This paper presented also an outlook to GSOC's near future plan of deploying control room systems virtually. This step has already been taken in other businesses. It will solve the topic of providing flexible systems while maintaining strict configuration control. It also approaches the current effect that control rooms are blocked by single projects for periods of one year or more for missions that take only a few weeks. 


\section{Acknowledgments}

The experiences shown here were made possible with the help of several more of our colleagues. The authors would like to thank Mr Gerd Enderlein and Mr Manfred Schmid for their support and consultancy.

\section{References}

Proceedings

${ }^{1}$ C. Haddow, “ESOC Ground Segments - The Future?”, AIAA 2008-3415, SpaceOPS, 2008

${ }^{2}$ Knorr, E. , Schmidhuber M., "Server Consolidation and Client Flexibility in GSOC Multimission Environment” RCSGSO 8 Workshop, 2009

${ }^{3}$ Navarro, V., "Virtualisation at ESOC” RCSGSO 8 Workshop, 2009

Reports, Theses, and Individual Papers

${ }^{4}$ Uschold, A., "Analyse der Virtualisierungsmöglichkeiten der IT-Infrastruktur für den Missionsbetrieb am deutschen Raumfahrtkontrollzentrum” Student Thesis, Chair of Aeronautical Engineering., Technical University, Munich, 2008.

Computer Software

${ }^{5}$ VMWare Player, Ver. 2.5.2, vmware Inc., 2009, URL: http://www.vmware.com.

${ }^{6}$ VMWare ESXi Server, Ver. 4.0, vmware Inc., 2009, URL: http://www.vmware.com. 